\title{
Detached chip smoothing during involute gear slotting in relation to generated tooth features
}

\author{
Mihai Bordeanu, Gabriel Frumuşanu* and Nicolae Oancea \\ Dunărea de Jos University of Galaţi, Department of Manufacturing Engineering, Domnească Street \\ 111, 800201 - Galaţi, Romania
}

\begin{abstract}
The problematic concerning the generating process uniformity, in the specific case of machining involute gear teeth with a rack shaped cutter provided by a single tooth is one of the notable challenges from gear teeth machining domain. In previous work, it has already been presented a solution of this problem, based on a graphical application in CATIA. The solution involves the modification of enveloping process ongoing, such as the circular feed motion takes place with variable speed. The variation law of feed motion speed that should be followed for smoothing the detached chip area is, obviously, different for each gear-type specific case. This paper is aiming to study the influence of generated tooth features onto this law form. Graphical and numerical applications addressing different cases of teeth numbers for the generated gear are presented and analyzed.
\end{abstract}

\section{Introduction}

The process of generating the tooth space of machined gear is characterized by high nonuniformity, which further leads to unwanted variation of cutting specific stress, depending on the relative position between tool and workpiece, with direct impact on the magnitude of main cutting force [1]. Research papers such as [2,3] analyze the toothing process deployment. The problem of finding the variation law for the energetic load of the rack shaped cutter when generating, by rolling method, the straight teeth of gears with involute profile has already been addressed in two different approaches concerning the toothing process smoothing by modifying the detached chip area. The first approach lies on changing the constructive shape of the rack-tool [4], while the second grounds on varying the rolling motion speed, according to an appropriate law, which enables to obtain a quasiconstant area of the detached chip [5]. The aspects of the problem defined and developed by [5] in the case of a mono-tooth rack shaped tool, which generates in a single pass the entire space between the flanks of two successive teeth, should be extended for gears having diverse numbers of teeth and, at the same time, to the situation when generating involute gears by multiple, successive passes. This can be done by appealing to the principles of generating surfaces by enwrapping [6-8], but also by taking advantage from the facilities of the graphical design environments, such as CATIA.

\footnotetext{
*Corresponding author: gabriel.frumusanu@ugal.ro
} 
This paper purpose is to study the influence of generated tooth features onto the variation law of the rolling motion speed. The next section is dedicated to the analytical modelling of tooth space generating with shifted position of mono-tooth rack shaped tool. The third section studies the variation of the detached chip area in three different cases, by graphical means. The fourth section deals with the analysis of the variation laws of the rolling motion speed, when a constant area of the detached chip is intended, while the last section is for conclusion.

\section{Analytical modelling of tooth space generating with shifted position of mono-tooth rack shaped tool}

The algorithm for finding the equations of the generated profile in the case when the rack shaped tool takes up a shifted position relative to its centrode is further presented.

\subsection{Equations of shifted tool tooth}

In Figure 1, there are presented the normal position of tool tooth, the centrodes, the reference systems and the specific kinematics of generating process (a), and the shifted position of tool tooth, measured by $\delta$ parameter $(b)$.

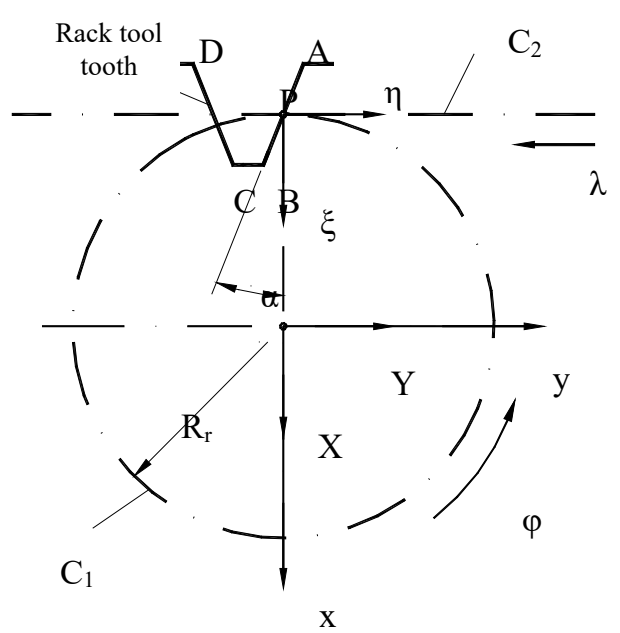

(a)

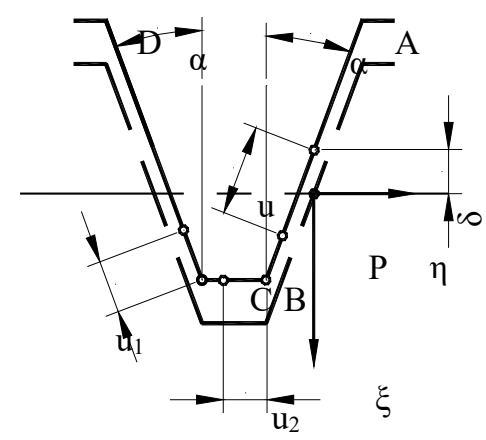

(b)

Fig. 1. Rack-tool tooth: (a) - Centrodes, motions \& reference systems (b) - Shifted profile.

The rolling motion takes place between the centrodes $C_{l}$ - circle of $\mathrm{R}_{\mathrm{r}}$ radius, having rotation motion of $\varphi$ angular parameter, associated to workpiece, and $C_{2}-$ a straight line tangent to $C_{l}$, in translation motion of $\lambda$ linear parameter, associated to the tool. The rolling condition between the two centrodes is cinematically expressed by:

$$
\lambda=R_{r} \cdot \varphi \text {. }
$$

Finding of the equation of the workpiece generated profile requires the following reference systems to be considered:

- $\quad x y$, which is the global system, having the origin in workpiece centre;

- $X Y$ - relative system, attached to the workpiece and initially overlapped to $x y$;

- $\quad \xi \eta$ - relative system, attached to the tool. 
The tool tooth profile is formed by the ensemble of the three straight segments, $\overline{A B}$, $\overline{B C}$ and $\overline{C D}$. Their equations, in the case of tool shifted position, result by expressing the coordinates of the current point belonging to each of them, as it follows:

$$
\begin{gathered}
\overline{A B} \mid \begin{array}{l}
\xi=-\delta+u \cos \alpha ; \\
\eta=-u \sin \alpha
\end{array} \\
\overline{B C} \mid \begin{array}{l}
\xi=-\delta+1.25 \cdot m \\
\eta=-1.25 \cdot m \sin \alpha-u_{2},
\end{array} \\
\overline{C D} \mid \begin{array}{l}
\xi=-\delta+u_{\max } \cos \alpha-u_{1} \cos \alpha ; \\
\eta=-u_{\max } \sin \alpha-u_{2 \max }-u_{1} \sin \alpha .
\end{array}
\end{gathered}
$$

In equations (2) - (4), $u, u_{1}$ and $u_{2}$ are linear parameters, $u_{\max }=u_{1 \max }=1.25 \cdot \mathrm{m} / \cos \alpha$, $u_{\text {min }}=u_{1 \min }=-1.25 \cdot m / \cos \alpha, \quad u_{2 \min }=0$ and $u_{2 \max }=m \cdot \pi / 2-2.5 \cdot m \sin \alpha, m$ meaning gear module.

\subsection{Profiles family}

The family of successive positions of tool tooth flanks, meaning the successive cuts of the rack shaped tool in the workpiece solid material results from the equation of relative motion between tool and workpiece:

$$
\left(\begin{array}{c}
X \\
Y
\end{array}\right)=\left(\begin{array}{cc}
\cos \varphi & \sin \varphi \\
-\sin \varphi & \cos \varphi
\end{array}\right)\left[\left(\begin{array}{l}
\xi \\
\eta
\end{array}\right)+\left(\begin{array}{c}
-R_{r} \\
-R_{r} \cdot \varphi
\end{array}\right)\right]
$$

If in relation (5), $\xi$ and $\eta$ are replaced with their calculus relations from (2), (3) and (4), then the equations of $\overline{A B}, \overline{B C}$ and $\overline{C D}$ segments family result, respectively. For sampling, the $\overline{A B}$ segment family equations are:

$$
\left(\Sigma_{A B}\right)_{\varphi} \mid \begin{aligned}
& X=\left(-\delta+u \cos \alpha-R_{r}\right) \cos \varphi-\left(u \sin \alpha+R_{r} \cdot \varphi\right) \sin \varphi \\
& Y=-\left(-\delta+u \cos \alpha-R_{r}\right) \sin \varphi-\left(u \sin \alpha+R_{r} \cdot \varphi\right) \cos \varphi
\end{aligned}
$$

A profiles family of (6) type (obviously, together to the ones corresponding to $\overline{B C}$ and $\overline{C D}$ ), in the case of an imposed value for shifting parameter $\delta$ (see figure 1-b) accepts an envelop, which represents the profile of the generated tooth space.

\subsection{Enveloping condition}

The enveloping condition may be written by choosing one of the several complementary theorems [8]. For example, according to Minimum distance method, the enveloping condition specific form is:

$$
\left[X-X_{P}\right] \cdot \dot{X}_{u}+\left[Y-Y_{P}\right] \cdot \dot{Y}_{u}=0
$$


In relation (7) $X$ and $Y$ are calculated with (6) (and its equivalents for $\overline{B C}$ and $\overline{C D}$ ), while $X_{P}$ and $Y_{P}$ mean the coordinates of gearing pole $P$ (see Figure 2):

$$
P: X_{P}=-R_{r} \cos \varphi ; Y_{P}=R_{r} \sin \varphi .
$$

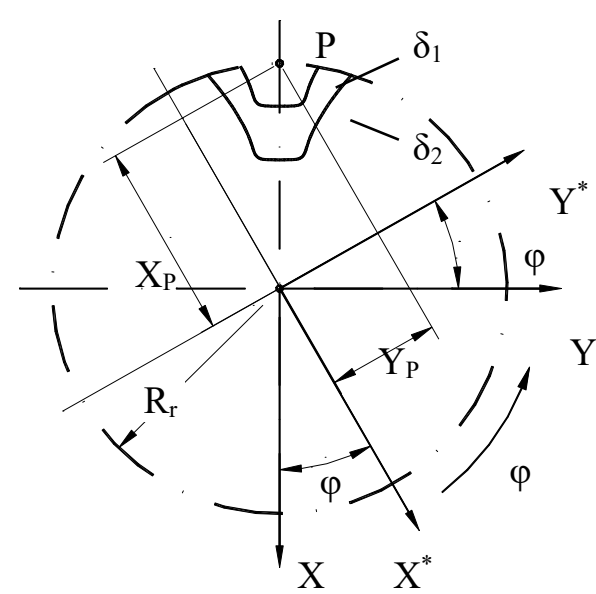

(a)

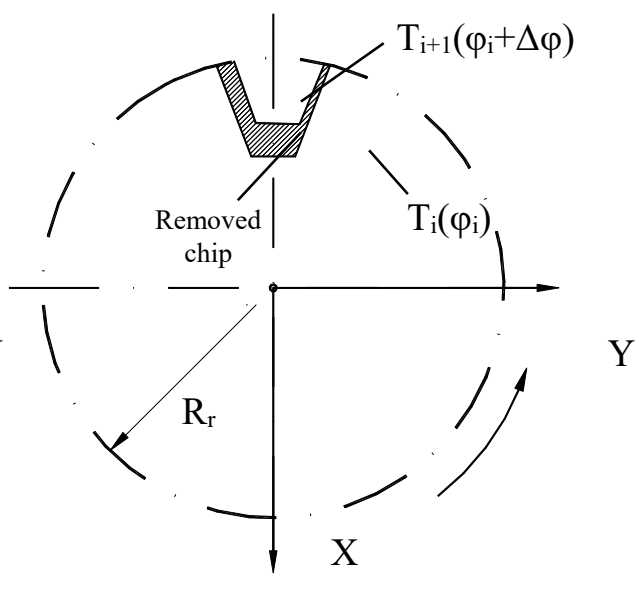

(b)

Fig. 2. Tooth space generation: (a) - Tooth space shape (b) - Successive cuts of tool tooth.

In Figure 2, $X^{*} Y^{*}$ represents the position of $X Y$ system after generated gear rotation by $\varphi$ angle. It should be noticed that different values of shifting parameter $\delta$ lead to different shapes of generated tooth space (see Figure 2-a), as stages in removing the entire volume of material from workpiece. In principle, the successive positions of rack tool (corresponding to $\Delta \varphi$ increment in workpiece's rotation motion) determine the shape and the area of removed chip (see Figure 2-b). The analytical solution of actually finding the relation between detached chip area and workpiece rotation angle is, obviously, laborious. This can be easier done by appealing to the facilities of CATIA graphical environment, as further suggested.

\section{Graphical modelling of tooth space generating}

The area of the chip detached at each active stroke of the rack shaped cutter can be found by graphical modelling the toothing process in CATIA. The method dedicated to this purpose, presented in [5], has been comparatively applied in three different cases of gears, having the same module, $m=10 \mathrm{~mm}$, teeth number being $z_{1}=30, z_{2}=45$ and $z_{3}=60$, hence the corresponding rolling radii $R_{r}$ were 150,225 and $300 \mathrm{~mm}$, respectively. The variation law of detached chip area, if machining with constant feed speed, has been found at first, in all three cases. In order to make the comparison relevant, the circular feed speed has been chosen different in each case, such as the maximum area of detached chip results similar (between $6-6.5 \mathrm{~mm}^{2}$ ). Thus, workpiece angular increments in the rolling motion were $\Delta \varphi_{1}=0.4, \Delta \varphi_{2}=0.35$ and $\Delta \varphi_{1}=0.3 \mathrm{deg} / \mathrm{dbl}$ stroke, respectively. The total numbers of required double strokes were 112, 98 and 96, respectively. The results are sampled, in numerical form, in Table 1 (detached chip areas for first 10 double strokes) and completely presented, in graphical form, in Figure 3. After that, the algorithm for cutting process smoothing [5] has been applied, in all three cases also, and the variation laws of the nonuniform circular feed (which always remains strictly correlated, according to condition (1), with rack shaped tool linear feed,) results as depicted in Figure 4. The maximum circular 
feed has been limited at $0.4 \mathrm{deg} / \mathrm{dbl}$. stroke from reasons in connection to the roughness of generated surface.

Table 1. Removed chips area.

\begin{tabular}{|c|c|c|c|c|c|c|}
\hline \multirow{2}{*}{$\begin{array}{c}\text { Crt. } \\
\text { no. }\end{array}$} & \multicolumn{2}{|c|}{$\mathbf{z}_{\mathbf{1}}=\mathbf{3 0}$ teeth } & \multicolumn{2}{c|}{$\mathbf{z}_{\mathbf{2}}=\mathbf{4 5}$ teeth } & \multicolumn{2}{c|}{$\mathbf{Z}_{\mathbf{3}}=\mathbf{6 0}$ teeth } \\
\cline { 2 - 7 }$\left[\left[^{\circ}\right]\right.$ & $\begin{array}{c}\text { Chip area, } \\
\mathrm{A}_{\mathrm{c}}\left[\mathrm{mm}^{2}\right]\end{array}$ & $\begin{array}{c}\text { Angle, } \\
\varphi\left[{ }^{\circ}\right]\end{array}$ & $\begin{array}{c}\text { Chip area, } \\
\mathrm{A}_{\mathrm{c}}\left[\mathrm{mm}^{2}\right]\end{array}$ & $\begin{array}{c}\text { Angle, } \\
\varphi\left[{ }^{\circ}\right]\end{array}$ & $\begin{array}{c}\text { Chip area, } \\
\mathrm{A}_{\mathrm{c}}\left[\mathrm{mm}^{2}\right]\end{array}$ \\
\hline 1 & 0 & 0.000 & 0 & 0.000 & 0 & 0.000 \\
\hline 2 & 0.3 & 0.923 & 0.35 & 0.492 & 0.4 & 0.376 \\
\hline 3 & 0.6 & 1.920 & 0.7 & 1.462 & 0.8 & 1.115 \\
\hline 4 & 0.9 & 2.947 & 1.05 & 2.406 & 1.2 & 1.836 \\
\hline 5 & 1.2 & 3.948 & 1.4 & 3.330 & 1.6 & 2.534 \\
\hline 6 & 1.5 & 4.932 & 1.75 & 4.227 & 2 & 3.214 \\
\hline 7 & 1.8 & 5.525 & 2.1 & 5.108 & 2.4 & 3.877 \\
\hline 8 & 2.1 & 5.654 & 2.45 & 5.690 & 2.8 & 4.520 \\
\hline 9 & 2.4 & 5.771 & 2.8 & 5.825 & 3.2 & 5.153 \\
\hline 10 & 2.7 & 5.874 & 3.15 & 5.932 & 3.6 & 5.529 \\
\hline \multicolumn{7}{r}{} \\
\hline
\end{tabular}

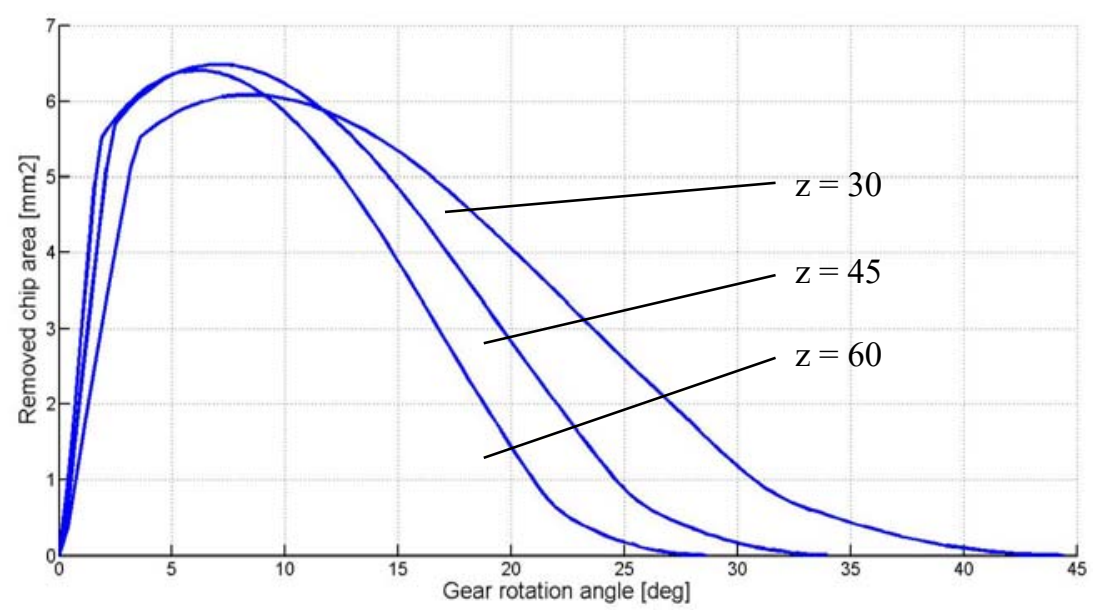

Fig. 3. The influence of gear teeth number $z$ on variation law of detached chip area.

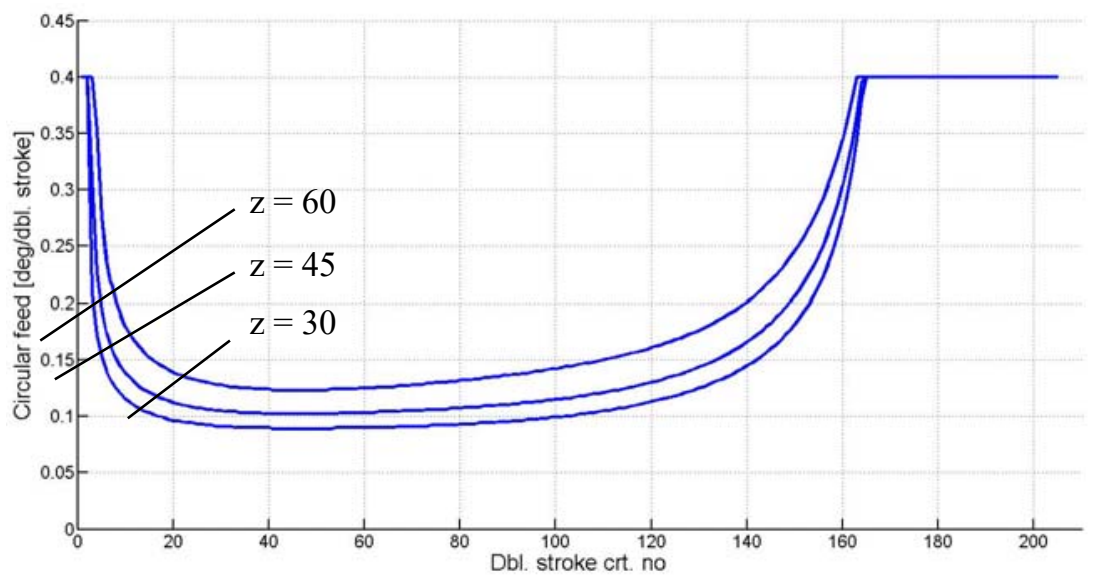

Fig. 4. Circular feed variation laws for uniform area of detached chip. 
If the feed variation law is modelled by a $8^{\text {th }}$ degree polynomial function expressing $\Delta \varphi$ value depending on dbl. stroke crt. no, $i$ :

$$
\Delta \varphi(i)=p_{1} \cdot i^{8}+p_{2} \cdot i^{7}+\ldots+p_{8} \cdot i+p_{9},
$$

Then the results obtained with Curve fitting tool from MatLab are presented in Table 2.

Table 2. Polynomial coefficients.

\begin{tabular}{|c|c|c|c|c|c|c|c|c|c|}
\hline $\begin{array}{c}\mathbf{Z} \\
\text { [teeth] }\end{array}$ & $\mathbf{p}_{1}$ & $\mathbf{p}_{2}$ & $\mathbf{p} 3$ & $\mathbf{p} 4$ & $\mathbf{p} 5$ & $\mathbf{p} 6$ & $\mathbf{p}_{7}$ & $\mathbf{p} 8$ & $\mathbf{p 9}$ \\
\hline 30 & 5.883 & -3.88 & 1.069 & -1.58 & 1.36 & -6.91 & 0.0020 & -0.0298 & 0.3085 \\
& $\cdot 10^{-16}$ & $\cdot 10^{-13}$ & $\cdot 10^{-10}$ & $\cdot 10^{-8}$ & $\cdot 10^{-6}$ & $\cdot 10^{-5}$ & & & \\
\hline 45 & 8.314 & -5.58 & 1.56 & -2.34 & 2.03 & -10.32 & 0.0029 & -0.0418 & 0.3328 \\
& $\cdot 10^{-16}$ & $\cdot 10^{-13}$ & $\cdot 10^{-10}$ & $-10^{-8}$ & $\cdot 10^{-6}$ & $\cdot 10^{-5}$ & & & \\
\hline 60 & 8.441 & -5.63 & 1.558 & -2.31 & 1.98 & -9.91 & 0.0027 & -0.0376 & 0.2830 \\
& $\cdot 10^{-16}$ & $\cdot 10^{-13}$ & $\cdot 10^{-10}$ & $\cdot 10^{-8}$ & $\cdot 10^{-6}$ & $\cdot 10^{-5}$ & & & \\
\hline
\end{tabular}

As it can easily be noticed, the variation laws presented in Figure 4 and the polynomial coefficients values look very similarly, which entitles us to consider that same feed variation law might be used when machining gears having the same module, possibly after applying a correction coefficient depending on teeth number.

\section{Conclusion}

This paper analyzes the applicability of the method for smoothing the energetic load along the rack tool cutting edge, developed by the same researchers collective and presented in a previous paper. More precise, the analytical modelling for teeth generating with shifted position of rack shaped tool has been realized in order to open the gate towards a more convenient distribution of material to be removed during tooth generating process. At the same time, the possibility of using the same basic feed variation law for more than a single actual case of gear machining, which may lead to a simpler programming of the machine tool, has been investigated and the results are promising. The remaining challenges are to extend the application domain to other types of tools \& generated surfaces and to develop a control system enabling to actually implement in practice the imagined solution.

\section{References}

1. J. Ma, X. Ge, S. I. Chang and S. Lei, Int J Adv Manuf Technol, 74, pp.1701-1708, (2014)

2. A. Antoniadis, N. Vidakis and N. Bilalis, J Manuf Sci Eng, 124, pp.784-791, (2002)

3. V. Georgescu, N. Oancea and E. Constantin, Buletinul Universităţii din Galaţi, Fasc. V, pp.33-42, (1982)

4. M. Dima and N. Oancea, Romanian Journal of Technical Sciences, Applied Sciences, 49, pp.237-241, (2004)

5. M. Bordeanu, G. Frumusanu and N. Oancea, MATEC Web of Conferences, 112, 01008, DOI: 10.1051/matecconf/201711201008, (2017)

6. S. P. Radzevich, Kinematics Geometry of Surface Machining (CRC Press, London, 2008)

7. F. L. Litvin, Theory of gearing (NASA, Scientific and Technical information Division, Washington DC, 1984)

8. N. Oancea, Surface Generation by Enwrapping, Vol. II, Complementary theorems (,Dunărea de Jos” Publishing House, Galaţi 2004) 